\title{
Health literacy, culture and Pacific peoples in Aotearoa, New Zealand: A review
}

\author{
Losi Sa'u Lilo, El-Shadan Tautolo, Melody Smith
}

\begin{abstract}
The social and cultural determinants of health among Pacific people must be addressed to understand the underlying factors related to poor health outcomes. Such factors may include (but are not be limited to) culture, religion, education, socioeconomic status and health literacy. One study, using the lens of Pacific culture, found that almost $90 \%$ of Pacific males and females aged 15 years and over have low health literacy. Individuals with low health literacy are less likely to manage ill health, seek professional medical assistance or interpret nutrition related information. It is possible that the high rates of non-communicable diseases (NCDs) as a significant issue in the Pacific population, including amongst Pacific mothers, are in some part associated with low levels of health literacy, which in turn link to cultural determinants of health. Findings from this review show that inadequate health literacy was consistent among adult females, particularly older adults of low socioeconomic status, lower level of education, non-English speakers and adults with compromised health status. Further, culture may play a role in attainment of adequate health literacy. These individuals were more likely to report worse chronic physical conditions, such as diabetes, including lack of knowledge of their condition such as the inability to identify normal blood sugar levels, the range of a normal blood pressure or how to self-manage hypoglycaemia. Public health practitioners should apply effective communication using a culturally and ethnically tailored approach to support Pacific peoples to understand health messages, improve health behaviours and health status. The author reviewed 33 papers on the issue of health literacy definitions, measurement and determinants; Pacific peoples and NCDs; and discussed it in the light of a cultural determinants' approach.
\end{abstract}

Keywords: health literacy, Pacific peoples, NCDs, health and culture

Losi Sa'u Lilo is a PhD Candidate and Researcher in the Centre for Human Potential, Auckland University of Technology (AUT). Correspondence 1saulilo@aut.ac.nz.

El-Shadan Tautolo is Director of AUT Centre for Pacific Health and Development Research, Faculty of Health \& Environmental Sciences, AUT University.

Melody Smith is Associate Head (Research) at the School of Nursing, University of Auckland. 


\section{Introduction}

Pacific peoples in New Zealand experience an inequitable burden of noncommunicable diseases (NCDs). Pacific peoples in New Zealand consist of numerous Pacific ethnicities namely eight main Pacific ethnicities whereby Samoans comprise the largest Pacific ethnic group 49\%, followed by Cook Island Māori $(21 \%)$, Tongan (20\%), Niueans (8\%), Fijian (5\%), Tokelauan (2\%), Tuvaluan (1\%) and Kiribati $(0.7 \%)$ (Tukuitonga, 2013; Pasefika Proud, 2016). They are the fourth largest ethnic group in New Zealand, behind European, Māori and Asian ethnic groups (The Treasury, 2018, p. 9), mostly comprising of youth (55\%) aged under 25 years compared to $34 \%$ of New Zealanders aged under 25 years. Most Pacific Peoples $(92.9 \%$ or 274,806 people) live in the North Island and of these, $66 \%$ reside in Auckland, the largest city in New Zealand. Furthermore, almost two thirds $165.9 \%$ or 194,958 people) identified with at least one Pacific ethnicity and lived in the Auckland region; and $12.2 \%$ or 36,105 people reside in the Wellington region. In contrast only $7.1 \%$ of Pacific Peoples (21,135 people) live in the South Island (Stats NZ, 2013). It is estimated that by 2038 Pacific peoples will make up $10.9 \%$ of the total New Zealand population (Pasefika Proud, 2016). The contributions that Pacific peoples make to New Zealand's society, economy and identity continues to form an increasingly important part of its future (The Treasury, 2018, p. 4). However, poor health outcomes among Pacific populations in New Zealand needs significant improvement (MoHNZ, 2019).

Pacific adults in New Zealand have the highest prevalence of NCDs compared with their non-Pacific counterparts. An estimated $66.9 \%$ of Pacific adults are obese compared to $32 \%$ of the total New Zealand population (MoHNZ, 2019; The Treasury, 2018 , p. 9). NCDs are classed as medical conditions or diseases which are noninfectious, non-transmissible, and in many cases are preventable (MoHNZ, 2019). Major NCDs comprise obesity, cardiovascular disease (CVD), heart disease, stroke, high blood pressure and some cancers (MoHNZ, 2019). In New Zealand the leading cause of mortality is CVD followed by diabetes and thirdly stroke (MoHNZ, 2019). New Zealand mortality rates continue to increase due to diabetes, cancer, and respiratory conditions with Pacific adults and children having the highest rates of obesity (MoHNZ, 2019). Evidence shows that behavioural factors such as physical inactivity, poor diet and smoking are associated with poor health among Pacific adults (MoHNZ, 2019; Stats NZ, 2013). Identifying the most common underlying causal factors for detrimental health is important as this could aid in identifying more effective health interventions or preventable measures for ill health (Kickbusch, 2001). Social and cultural determinants of health need to be addressed in order to understand the underlying factors related to poor health status. Such factors may include (but are not be limited to) culture, religion, education, socioeconomic status (Braveman et al., 2011; MoHNZ, 2012) and health literacy (Kickbusch, 2001; Kutner et al., 2006; Schillinger et al., 2002).

One factor that may contribute to the inequitable burden of NCDs in Pacific people is low health literacy. One study found almost $90 \%$ of Pacific males and females aged 15 years and over have low health literacy (MoHNZ, 2012; University of Otago, \& MoHNZ, 2011). Health literacy is defined as the ability to access, read, understand and interpret basic health related information to make informed health decisions to improve health outcomes (Kickbusch, Maag \& Saan, 2005). Individuals with low health literacy are less likely to manage ill health including diabetes (Harris, 2000; Kim, Love, Quistberg \& Shea, 2004), seek professional medical assistance (World Health Organisation [WHO], 2019), or interpret nutrition related information (U.S. Department of Health and Human Services, 2005).

The aim of this literature review is to identify relationships between health literacy and health outcomes with a particular focus on primary underlying social factors 
faced by Pacific people (such as culture, religion, and socioeconomic status). In doing so, this review will examine the public health problem of NCD prevalence among the Pacific population in New Zealand, investigating health literacy, within a social determinants and particularly cultural determinants lens, as a potential means of managing and preventing NCDs for good health outcomes of the Pacific population.

Literature was identified from the following databases: PubMed, Google scholar and EBSCOhost as well as government agency websites including the Ministry of Health New Zealand, Ministry of Education New Zealand, and the World Health Organisation. The search was conducted between March 2013 and June 2013, further updated from Jan 2016 to April 2019 and included a total of 26 articles, published from 1998 to 2019. The following search terms were used: nutrition, health literacy, socioeconomic status, health outcomes, adults, research, culture, diabetes, and obesity. Peer reviewed scholarly journal articles and key reports relating to NCDs among Pacific peoples within New Zealand and studies from international countries were included; thus 26 papers are presented and discussed.

\section{Health Literacy as a potential factor associated with Pacific health status}

\section{Definitions of Health Literacy}

No agreed upon definition of health literacy exists due to its' complexity and multifaceted nature (Berkman, Davis, McCormack, 2010; Kickbusch, 2001). Health literacy has been defined as the ability to read, understand and interpret health information to better enhance individual health status (Berkman, Davis, \& McCormack, 2010). In a similar vein, the New Zealand Ministry of Health (2010) defined health literacy as the degree to which individuals have the capacity to obtain, process and understand basic health information and services in order to make informed and appropriate health decisions' (pp. 1). The New Zealand Ministry of Health more recently defined health literacy as the "capacity to find, interpret and use information and health services to make effective decisions for health and wellbeing" (MoHNZ, 2015). In this definition the focus is most obviously on consumer capability. However, internationally support is growing for a stronger focus on how health systems, health care providers and practitioners who can support consumers to access and understand health services, and an appreciation for the factors which increase vulnerability of populations to poor health inhibiting health decision making (Commission on Social Determinants of Health, 2008).

The WHO definition states that healthy literacy is

"the cognitive and social skills which determine the motivation and ability of individuals to gain access to, understand and use information in ways which promote and maintain good health for themselves, their families and their communities. Health literacy means more than being able to read pamphlets and successfully make appointments. By improving people's access to health information and their capacity to use it effectively, health literacy is critical to empowerment. Health literacy implies the achievement of a level of knowledge, personal skills and confidence to take action to improve personal and community health by changing personal lifestyles and living conditions" (as cited in Nutbeam, 1998).

It goes on to say that health literacy at a population level yields social benefits such as mobilizing communities to address the social, economic and environmental determinants of health. Thus in part, equal attention is to be provided from governments and health systems to present clear, accurate, appropriate and 
accessible information for diverse audiences rather than the sole responsibility falling on the individual (WHO, 2019).

\section{Measures of Health Literacy}

The lack of consistency in defining health literacy is just as complex as the measures of health literacy (Nutbeam, 2000). There are various measures used to capture levels of health literacy: the rapid estimate of adult literacy in medicine (REALM); the test of functional health literacy in adults (TOFHLA); and the short test of functional health literacy in adults (S-TOFHLA) (Baker, Williams, Parker, Gazmararian, \& Nurss, 1999) are commonly used; also the single item literacy screener (SILS) (Morris, MacLean, Chew, \& Littenberg, 2006). The REALM test only measures the ability to pronounce words in isolation and little is known about the relationship of the REALM to patients' self-management skills, health and use of health care services (Baker et al., 1999). In addition, REALM ( $\mathrm{n}=66$ items) appears to have both overestimated and underestimated patients' reading ability when compared to the s-TOFHLA ( $\mathrm{n}=$ 36 items). The TOFHLA was considered the best form of measuring health literacy since the early 1990s but in 1998 the s-TOFHLA was introduced as a more reliable and valid instrument (Baker et al., 1999). The s-TOFHLA is used to assess a patient's health literacy level based on reading comprehension and numeracy skills (Baker et al., 1999). When created, the number of questions ( $n=36$ items) was reduced for the $\mathrm{s}$-TOFHLA but included similar health related questions as the TOFHLA ( $\mathrm{n}=67$ items). The intention was to reduce the time frame to complete questions so that numeracy and reading comprehension results were measured with precision (Baker et al., 1999). Validity and reliability of literacy and numeracy skills was measured using the Cronbach's alpha (r); a common form of reliability testing in statistics to determine insignificant or significant internal consistency between two measures (Bland \& Altman, 1997). Good internal consistency is classified as $r>0.80$ whereas $r<0.70$ indicates questionable-to-insignificant correlations (Bland \& Altman, 1997). For example, when s-TOFHLA was compared to REALM the internal consistency resulted in 0.80 (Baker et al., 1999). Further, internal consistency for reading comprehension was 0.97 and for numeracy skills 0.68 (Baker et al., 1999). A study conducted in an urban public hospital in Atlanta, US among African American adult patients, compared scores between s-TOFHLA, REALM and TOFHLA, found excellent reliability and validity across the different tools (Baker et al., 1999a). When compared to the REALM the internal consistency resulted in 0.80. Further, based on the reading passages, the internal consistency measured 0.97 between the REALM and s-TOFHLA and 0.68 for numeracy skills.

The s-TOFHLA questionnaire includes four numeracy items with a potential sevenpoint score per question answered correctly and the reading comprehension passages cover 36 questions with a two-point score per question. Reading comprehension questions cover health related sentences such as: "The x-ray will take from 1 to 3 hours to do". The fifth or ninth word in each sentence is then deleted and the best suited word must be inserted. A list of words is available for selection of the appropriate word to insert. Numeracy questions include passages such as: "If your blood sugar score is 160 , would your blood sugar be normal?". Scores from 0 to 53 indicates inadequate or low health literacy; a score from 54 to 66 indicates marginal health literacy and a score of 67 to 100 shows adequate or good health literacy (Baker et al., 1999).

The s-TOFHLA has the ability to identify an individual's inadequate functional health literacy within a 10 to 12 minute time frame compared to TOFHLA which requires a 22 minute completion period (Baker et., 1999). In addition, it is easier to administer than the full battery, and scoring is less subjective (Baker et al., 1998). Testing of the $\mathrm{s}$-TOFHLA suggests this is a reliable and valid measure of health literacy, while also 
being shorter to complete than the TOFHLA and REALM (Baker et al., 1999). Reliability testing of this measure has been conducted with small homogenous populations; testing in a larger, more diverse population is needed to confirm reliability (Baker et al., 1998).

SILS is a simple instrument, providing help with reading health-related materials, designed to identify patients with limited reading ability resulting from language barriers, low educational attainment and cultural norms (Morris et al., 2006). The SILS is used to assess a patient's health literacy level based on reading comprehension and numeracy skills (Morris et al., 2006). The SILS question asks: "How often do you need to have someone help you when you read instructions, pamphlets, or other written material from your doctor or pharmacy?" Participants can select from the following scale in response to this question including: 1-Never, 2-Rarely, 3-Sometimes, 4-Often, and 5-Always. Scores from responses 1 and 2 are classified as 0 (No) and scores greater than 2 ( 3 to 5 ) classified as 1 (Yes). The value 0 indicates 'good' or no difficulty with reading and interpreting health related information and responses for the value 1 indicated 'poor' or some difficulty with reading printed health related material (Morris et al, 2006).

Conclusively, these tools are useful for identifying patients for whom standard care approaches and materials are a barrier to managing their health. They have allowed researchers to measure reading skills in health care settings and subsequent treatment when literacy abilities have been identified and documented in medical care settings. Although, such tests cannot determine the cause, type of reading or learning difficulty and thus cannot diagnose specific problems (Davis, Michielutte, Askov, Williams, and Weiss, 1998). Further, researchers have not yet adequately explored the experience of patients taking these assessment tests, nor have they examined implications for patient dignity and subsequent treatment when literacy abilities are identified and documented in medical care settings (as sited in Rudd, Moeykens, \& Colton, 1999).

\section{Health Literacy and Pacific Culture}

Culture has been described as a defining feature or characteristic of Pacific people (Lui, 2003). According to Pulotu Endemann (2011) cultural values and beliefs are the shelter for life. The sharing of food is a fundamental cultural value in Pacific communities encompassing traditional sunday church gatherings as well as traditional celebrations such as weddings and funerals (Lui, 2003). Food plays a significant role in social and cultural Pacific settings over and above biological needs for nourishment and survival (Rush, 2009). In New Zealand, culture, family and personal attitudes influence the food choices of Pacific people (Stats NZ, 2013). Foods of lesser nutritional value containing high salt, sugar and saturated fat content are often consumed for their flavour and as their convenience influences the opportunity to purchase such foods (Health Partners Consulting Group, 2012). Such foods are often served and consumed at traditional cultural gatherings (Rush, 2009).

This situation is indicative of two possible scenarios related to health literacy and health behaviours. Firstly, Pacific communities may have health knowledge, and are able to identify the differences between healthy and unhealthy foods yet continue to consume unhealthy foods due to preference, cost, convenience and cultural practices. For example, despite the need for individual health attainment, it is culturally valued at cultural settings that the sharing and consuming of such foods occurs to show respect and common courtesy (Lui, 2003). The risks to health are significant as social gatherings occur frequently and foods are available in large amounts (Lui, 2003; Rush, 2009). Secondly, consuming such foods as part of cultural practices could indicate low health literacy in this population. 
Pacific Island families play a cultural role in providing for their respective families in New Zealand as well as their immediate or extended families living in the Pacific Island they originated from (Rush, 2009). Supporting their families in their country of birth financially is not only a cultural custom of Pacific populations but is the most common form of support, which adds to the financial burdens of New Zealand-based Pacific families (Rush, 2009). This then suggests that Pacific people in New Zealand are at a higher risk of low health literacy because of the influences of cultural values of non-native ethnic groups and low-income status which potentially eventuate in ill health outcomes.

\section{Health Literacy and Socioeconomic Status}

Low health literacy levels have also been associated with low socioeconomic status (Ishikawa \& Yano, 2008; Kim et al., 2001; Marks, Schectman, Groninger, \& PlewsOgan, 2010; McCleary-Jones, 2011; Sarkar, Liu, Moffet, \& Schillinger, 2010; Schillinger et al., 2002). Five studies (Kim et al., 2001; Marks et al., 2010; McClearyJones, 2011; Sarkar et al., 2010; Schillinger et al., 2002) found that low health literacy was common among women in the US who experienced NCDs, who were aged 55 to 90 years, and of low socioeconomic status (annual income less than USD $\$ 20,000$ ). These findings were consistent across differing health problems (e.g., type 2 diabetes, cancer). Few studies have shown associations between socioeconomic status and health literacy in males (Kim et al., 2001; Marks et al., 2010; McCleary-Jones, 2011; Sarkar et al., 2010; Schillinger et al., 2002). These may be a consequence of higher reported socioeconomic status, wider age ranges and smaller sample sizes for males than females in these studies. Most of the studies included males aged from 30 to 90 years and samples were smaller (i.e., a maximum of 20 males compared to 45 females) (Kim et al., 2001; McCleary-Jones, 2011; Sarkar et al., 2002).

Studies specifically of Pacific peoples from different populations are needed to measure differences by socio-demographic factors such as place of origin, sex, age, socio-economic status, and educational attainment. Using this approach could possibly show relatively comparable results of health literacy across, say for example, all age groups (McCleary-Jones, 2011; Sarkar et al., 2002; Schillinger et al., 2002). This is important because younger age groups and males could be overestimated as having adequate health literacy but if included in future research could show they are just as at higher risk as older adults and females. Such evidence could enhance current health literacy initiatives by offering evidence for better targeting.

Low health literacy has also been associated with those of middle to high socioeconomic status (Hesketh et al., 2005). An investigation of urban Australian mothers and their children of perceptions of nutrition, physical activity, and obesity provided some evidence of this (Hesketh et al., 2005). Mothers of middle to high income believed their children understood which foods were healthy and yet the mothers did not fully translate this in their own behaviours. This was reflected in the perceptions of their children whereby most children identified some unhealthy foods as healthy foods or were confused as to which types of foods to consume for a healthy lifestyle and what made up appropriate portion sizes. Leading a healthy lifestyle through healthy eating behaviours and being physically active were perceived as important factors and well understood by all mothers, but it was hard for them to translate this general knowledge into specific behaviours. However, this may reflect the complexity of nutritional information for the consumer as much as health literacy. It is likely that across all socioeconomic levels, inadequate health literacy is associated with inadequate management of ill health (Kim et al., 2001; Marks, Schectman, Groninger \& Plews-Ogan, 2009; McCleary-Jones, 2011; Sarkar et al., 2002; Schillinger et al., 2002) as well as perceived mixed messages around healthy 
lifestyles (Hesketh et al., 2005). Based on these studies, focusing on reducing socioeconomic inequalities and improving health literacy for all should remain a focal point of health policy and practice.

\section{Health Literacy and Health Outcomes}

Research to date shows that health literacy is associated with the effective management of NCDs including type 2 diabetes (Harris, 2000; Kim, Love, Quistberg, \& Shea, 2004; Sarkar et al., 2010; Schillinger et al., 2002; Williams, 1998b), asthma (Mancuso \& Rincon, 2006; Williams et al., 1998a), mental health (Jorm, 2000), prostate cancer (Kim et al., 2001), and obesity (Carmona, 2005). Three research studies showed associations between adults with inadequate health literacy and type 2 diabetes (Kim et al., 2001; Schillinger et al., 2002; Williams et al., 1998b). Findings showed inadequate health literacy was common in adults aged 45 years and older. Further, adults were less likely to self-manage their diabetes resulting in poor glycaemic control. They had higher prevalence of retinopathy (damage to the retina caused by type 2 diabetes) and increased likelihood of reporting complications of diabetes (Schillinger et al., 2002) compared to patients with marginal or adequate health literacy (William et al., 1998b).

Significant associations have also been found between inadequate health literacy and sub-optimal asthma control (Mancuso \& Rincon, 2006; Williams et al., 1998a), prostate cancer (Kim et al., 2001), and mental health (Jorm, 2000). The following common characteristics were associated with inadequate health literacy; patients were predominantly older females, spoke English as a second language, and had a level of education from high school or lower (Jorm, 2000; Kim et al., 2001; Mancuso \& Rincon., 2006; Williams et al., 1998b).

Carmona (2005) suggested individuals require further strategies to improve health literacy, ones which are tailormade, to avoid obesity based on traditional family customs of consuming foods high in saturated fats, salt and sugar. The researcher noted that American children and adults continue to engage in such lifestyles which inhibits their ability to reduce obesity rates (Carmona, 2005). Similar trends for Pacific populations exist thus there is a need to tailor communication to suit Pacific traditional ways of leading a healthy lifestyle to reduce morbidity and mortality associated with poor health outcomes (Health Partners Consulting Group, 2012).

\section{Recommendations}

The studies presented here indicate that inadequate health literacy is consistent among adult females, particularly older adults of low socioeconomic status (Kim et al, 2001; Marks et al., 2010; McCleary-Jones, 2011; Schillinger et al, 2002); with lower levels of education (McCleary-Jones, 2011; Schillinger et al, 2002); among nonEnglish speakers (Kim et al, 2001; Sarkar et al, 2010); and adults with compromised health status (Harris, 2000; Kim et al, 2004; Sarkar et al, 2012; Schillinger et al, 2002; William et al, 1998). Further, culture may play a role in attainment of adequate health literacy (Shaw, Huebner, Armin, Orzech, \& Vivian, 2009). Individuals with low levels of health literacy were more likely to report worse chronic physical conditions including lack of knowledge of their condition such as, in the case of diabetes, the inability to identify normal blood sugar levels, the range of a normal blood pressure or how to self-manage hypoglycaemia. However, most of the research has been conducted in settings other than Pacific communities, and there is a need for more research relating to such contexts. 


\section{Conclusion}

As previously discussed in this review, Pacific people living in New Zealand are more likely to have NCDs including type 2 diabetes, CVD, and obesity compared to all other ethnic groups in New Zealand. The most common influential risk factors for these diseases are unhealthy eating, lack of physical inactivity, and smoking. These factors are likely to be influenced by culture, religion, low socioeconomic status, low educational attainment, and low health literacy.

Social, economic and cultural determinants are potential threats to health especially for those with limited health literacy. The challenging role for public health practitioners is the ability to apply contextually appropriate strategies as an effective means to improving health literacy. Effective communication using a culturally and ethnically tailored approach is required that can help individuals add to their health literacy skills to understand health messages, improve health behaviours, health status and ultimately reducing morbidity and mortality. Any strategies addressing health literacy need to take place within a health system framework that addresses poverty and health equity.

\section{Acknowledgements}

Funding for this research was provided by Auckland University of Technology (AUT) Pacific Islands Families Study Masters Research Scholarship in support of LS. MS is supported by a Health Research Council of New Zealand Sir Charles Hercus Research Fellowship (grant number $17 / 013)$. 


\section{References}

Baker, D. W., Parker, R. M., Williams, M. V., \& Clark, W. S. (1998). Health literacy and the risk of hospital admission. Journal of general internal medicine, 13(12), 791-798. https://doi-org/10.1046/j.1525-1497.1998.00242.x

Baker, D. W., Williams, M. V., Parker, R. M., Gazmararian, J. A., \& Nurss, J. (1999). Development of a brief test to measure functional health literacy. Patient education and counseling, 38(1), 33-42. https://doi.org/10.1016/S0738-3991(98)00116-5

Berkman, N. D., Davis, T. C., \& McCormack, L. (2010). Health literacy: What is it? Journal of Health Communication, 15(S2), 9-19. https://doiorg.ezproxy.aut.ac.nz/10.1080/10810730.2010.499985

Berkman, N. D., Sheridan, S. L., Donahue, K. E., Halpern, D. J., \& Crotty, K. (2011). Low health literacy and health outcomes: an updated systematic review. Annals of internal medicine, 155(2), 97-107. https://doi.org/10.7326/0003-4819-155-2-20110719000005

Bland, J. M., \& Altman, D. G. (1997). Statistics notes: Cronbach's alpha. Bmj, 314(7080), 572. https://doi.org/10.1136/bmj.314.7080.572

Commission on Social Determinants of Health. (2008). Closing the gap in a generation: health equity through action on the social determinants of health. Final report of the Commission on the Social Determinants of Health. Geneva, World Health Organisation

Gary, T. L., McGuire, M., McCauley, J., \& Brancati, F. L. (2004). Racial comparisons of health care and glycemic control for African American and white diabetic adults in an urban managed care organization. Disease Management, 7(1), 25-34. https://doi.org/10.1089/109350704322918970

Harris, M. I. (2000). Health care and health status and outcomes for patients with type 2 diabetes. Diabetes Care, 23(6), 754-758. https://doi.org/10.2337/diacare.23.6.754

Health Partners Consulting Group. (2012). Metro-Auckland population health profile. Retrieved from http://www.health.govt.nz/publication/metro-auckland-pacificpopulation-health-profile

Hesketh, K., Waters, E., Green, J., Salmon, L., \& Williams, J. (2005). Healthy eating, activity and obesity prevention: a qualitative study of parent and child perceptions in Australia. Health promotion international, 2O(1), 19-26. https://doi.org/10.1093/heapro/dah503

Jorm, A. F. (2000). Mental health literacy: Public knowledge and beliefs about mental disorders. The British Journal of Psychiatry, 177(5), 396-401. https://doi.org/10.1192/bjp.177.5.396

Kim, S., Love, F., Quistberg, D. A., \& Shea, J. A. (2004). Association of health literacy with self-management behavior in patients with diabetes. Diabetes Care, 27(12), 29802982. https://doi.org/10.2337/diacare.27.12.2980

Kim, S. P., Knight, S. J., Tomori, C., Colella, K. M., Schoor, R. A., Shih, L., Bennett, C. L. (2001). Health literacy and shared decision making for prostate cancer patients with low socioeconomic status. Cancer investigation, 19(7), 684-691. https://doi.org/10.1081/CNV-100106143

Kickbusch, I. S. (2001). Health literacy: addressing the health and education divide. Health Promotion International, 16(3), 289-297. https://doi.org/10.1093/heapro/16.3.289

Lui, D. (2003). Family - A Samoan perspective. Retrieved from http://www.hdc.org.nz/publications/other-publications-from-hdc/mental-healthresources/family-a-samoan-perspective 
Mancuso, C. A., \& Rincon, M. (2006). Impact of health literacy on longitudinal asthma outcomes. Journal of general internal medicine, 21(8), 813-817. https://doi.org/10.1111/j.1525-1497.2006.00528.x

Marks, J. R., Schectman, J. M., Groninger, H., \& Plews-Ogan, M. L. (2010). The association of health literacy and socio-demographic factors with medication knowledge. Patient education and counseling, 38(3), 372-376. https://doi.org/10.1016/j.pec.2009.06.017

McCleary-Jones, V. (2011). Health literacy and its association with diabetes knowledge, selfefficacy and disease self-management among African Americans with diabetes $\begin{array}{lllll}\text { mellitus. } & A B N F & \text { Journal, } 2(2) . & \text { Retrieved from }\end{array}$ http://eds.a.ebscohost.com.ezproxy.aut.ac.nz

Ministry of Health. (2010). Ko rero $\mathrm{Ma}^{-}$rama: Health Literacy and $\mathrm{Ma}^{-}$ori Results from the 2006 Adult Literacy and Life Skills Survey. Wellington: Ministry of Health.

Ministry of Health. (2012). Tupu Ola Moui: Pacific health chart book 2012. Retrieved from http:/ / www.health.govt.nz/publication/tupu-ola-moui-pacific-health-chart-book2012

Ministry of Health. (2019). Annual Data Explorer 2017/18: New Zealand Health Survey [Data File]. URL: https://minhealthnz.shinyapps.io/nz-health-survey-2017-18annual-data-explorer

Morris, N. S., MacLean, C. D., Chew, L. D., \& Littenberg, B. (2006). The Single Item Literacy Screener: evaluation of a brief instrument to identify limited reading ability. BMC Family Practice, 7(1), 1. https://doi.org/10.1186/1471-2296-7-21

Nutbeam, D. (2000). Health literacy as a public health goal: a challenge for contemporary health education and communication strategies into the 21 st century. Health promotion international, 15(3), 259-267. https://doiorg.ezproxy.aut.ac.nz/10.1093/heapro/15.3.259

Oliver, M., Schluter, P. J., Paterson, J., Kolt, G. S., \& Schofield, G. M. (2009). Pacific Islands Families: Child and Parental Physical Activity and Body Size--design and methodology. The New Zealand Medical Journal (Online), 122(1298). Retrieved from URL: http://www.nzma.org.nz/journal/122-1298/3687/

Pasefika Proud. (2016). The Pacific Peoples Profile in New Zealand. Retrieved from http:/ / www.pasefikaproud.co.nz/assets/Resources-fordownload/PasefikaProudResource-Pacific-peoples-paper.pdf

Pulotu-Endemann, F. K. (2009). Fonofale Model of Health. Retrieved from http:/ / www.hauora.co.nz/resources/Fonofalemodelexplanation.pdf

Rudd, R. E., Moeykens, B. A., \& Colton, T. C. (1999). Health and literacy: a review of medical and public health literature. Office of Educational Research and Improvement (Online), (1), 1-41. Retrieved from https://files.eric.ed.gov/fulltext/ED508707.pdf

Rush, E. (2009). Food security for Pacific peoples in New Zealand: A report for the obesity action coalition. Retrieved from http://www.ana.org.nz/sites/default/files/PacificfoodsecurityreportfinalMarch09_3. pdf

Sarkar, U., Liu, J. Y., Moffet, H. H., \& Schillinger, D. (2010). Hypoglycemia is more common among type 2 diabetes patients with limited health literacy: the Diabetes Study of Northern California. Journal of general internal medicine, 25(9), 962-968. https://doi.org/10.1007/s11606-010-1389-7 
Schillinger, D., Grumbach, K., Piette, J., Wang, F., Osmond, D., Daher, C., Bindman, A. B. (2002). Association of health literacy with diabetes outcomes. JAMA: the journal of the American Medical Association, 288(4), 475-482. http://doi:10.1001/jama.288.4.475

Schillinger, D., Barton, L. R., Karter, A. J., Wang, F., \& Adler, N. (2006). Does literacy mediate the relationship between education and health outcomes? A study of a low-income population with diabetes. Public health reports, 121(3), 245-254. Retrieved from https:// doi.org/pdf/10.1177/003335490612100305

Shaw, S. J., Huebner, C., Armin, J., Orzech, K., \& Vivian, J. (2009). The Role of Culture in Health Literacy and Chronic Disease Screening and Management Journal of Immigrant and Minority Health, 11(6), 460-467. https://doi.org/10.1007/s10903-008-9135-5

Stats NZ. (2013). 2013 Census Group Profiles. Retrieved from http:/ / archive.stats.govt.nz/Census/2013-census / profile-and-summaryreports/ethnic-profiles.aspx

The Treasury. (2018). The New Zealand Pacific Economy. Retrieved from https://treasury.govt.nz/publications/commissioned-report/new-zealand-pacificeconomy

Williams, M. V., Baker, D. W., Honig, E. G., Lee, T. M., \& Nowlan, A. (1998a). Inadequate literacy is a barrier to asthma knowledge and self-care. CHEST Journal, 114(4), 10081015. https://doi:10.1378/chest.114.4.1008

Williams, M. V., Baker, D. W., Parker, R. M., \& Nurss, J. R. (1998b). Relationship of functional health literacy to patients' knowledge of their chronic disease: a study of patients with hypertension and diabetes. Archives of internal medicine, 158(2), 166. https://doi:10.1001/archinte.158.2.166

Wolf, M. S., Gazmararian, J. A., \& Baker, D. W. (2007). Health literacy and health risk behaviors among older adults. American journal of preventive medicine, 32(1), 19-24. https://doi.org/10.1016/j.amepre.2006.08.024

World Health Organisation. (2013). Health Literacy: The solid facts. Retrieved from http://www.euro.who.int/_data/assets/pdf_file/0008/190655/e96854.pdf

World Health Organisation. (2019). Health Literacy: The mandate for health literacy. Retrieved from literacy/en/ https://www.who.int/healthpromotion/conferences/9gchp/health- 